\title{
How Much Afterhyperpolarization Conductance Is Recruited by an Action Potential? A Dynamic-Clamp Study in Cat Lumbar Motoneurons
}

\author{
Marin Manuel, Claude Meunier, Maud Donnet, and Daniel Zytnicki \\ Neurophysique et Physiologie du Système Moteur, Unité Mixte de Recherche 8119, Centre National de la Recherche Scientifique, and Université René \\ Descartes, 75270 Paris Cedex 06, France
}

\begin{abstract}
We accurately measured the conductance responsible for the afterhyperpolarization (medium AHP) that follows a single spike in spinal motoneurons of anesthetized cats. This was done by using the dynamic-clamp method. We injected an artificial current in the neurons that increased the AHP amplitude, and we made use of the fact that the intensity of the natural AHP current at the trough of the voltage trajectory was related linearly to the AHP amplitude. We determined at the same time the conductance and the reversal potential of the AHP current. This new method was validated by a simple theoretical model incorporating AHP and hyperpolarization-activated $\left(I_{\mathrm{h}}\right)$ currents and could be applied when the decay time constant of the AHP conductance was at least five times shorter than the estimated $I_{\mathrm{h}}$ activation time. This condition was fulfilled in 33 of 44 motoneurons. The AHP conductance varied from 0.3 to $1.4 \mu \mathrm{S}$ in both slow- and fast-type motoneurons, which was approximately the same range as the input conductance of the entire population. However, AHP and input conductances were not correlated. The larger AHP in slow-type motoneurons was mainly attributable to their smaller input conductance compared with fast motoneurons. The likeness of the AHP conductance in both types of motoneurons is in sharp contrast to differences in AHP decay time and explains why slow- and fast-type motoneurons have similar gain.
\end{abstract}

Key words: discharge properties; afterhyperpolarizing current; synaptic integration; spinal cord; dynamic clamp; $I_{\mathrm{h}}$ current

\section{Introduction}

In many types of neurons, action potentials trigger an afterhyperpolarization (AHP) (Llinas and Yarom, 1981; Lancaster and Adams, 1986; Schwindt et al., 1988; Wilson et al., 2004). Different potassium currents underlie the fast, medium, and slow AHPs (Hounsgaard et al., 1988; Sah, 1996); they are, respectively, responsible for spike repolarization and early and late frequency adaptation. The medium AHP current is calcium dependent and mediated by small potassium (SK) channels (Barrett and Barrett, 1976; Schwindt and Crill, 1984; Zhang and Krnjevic, 1987). Medium AHP plays an important role in shaping the discharge properties. In particular, it sets the gain of spinal motoneurons (i.e., the slope of their current-frequency relationship) to low values (typically $1 \mathrm{~Hz} / \mathrm{nA}$ in anesthetized cats) and confines their steady discharge rate to the $10-50 \mathrm{~Hz}$ range. Reduction of the medium AHP by apamin or serotonin increases the gain and makes the discharge more irregular (Hounsgaard and Kiehn, 1989; Chandler et al., 1994; Lape and Nistri, 2000).

Received May 27, 2005; revised Aug. 8, 2005; accepted Aug. 10, 2005.

Financial support provided by Délégation Générale pour l'Armement (DGA) Grant 0034029 and by the Ministère de la Recherche (Action Concertée Incitative "Neurosciences Intégratives et Computationnelles") is gratefully acknowledged. M.M. is a DGA research fellow. We are indebted to Drs. Lyle Graham and David Hansel for useful comments and to Drs. Boris Lamotte d'Incamps and Alexander Roxin for careful reading of this manuscript.

Correspondence should be addressed to Dr. Daniel Zytnicki, Unité Mixte de Recherche 8119, Centre National de la Recherche Scientifique, 45 rue des Saints-Pères, 75270 Paris Cedex 06, France. E-mail: Daniel.Zytnicki@ univ-paris5.fr.

DOI:10.1523/JNEUROSCI.2154-05.2005

Copyright $\odot 2005$ Society for Neuroscience $\quad 0270-6474 / 05 / 258917-07 \$ 15.00 / 0$
Spinal motoneurons innervating slow twitch motor units have lower discharge frequency than those innervating fast units. This correspondence between the firing rate of motoneurons and the contractile properties of muscle fibers is mostly attributable to the medium AHP (Kernell, 1999). However, the relative impact of the conductance and relaxation time constant of the AHP current on the firing properties is not well understood. It is known that the medium AHP is longer in slow-type motoneurons. In contrast, it is unclear whether the AHP conductance is different in slow- and fast-type motoneurons. The present work addresses this issue.

To determine differences in AHP conductance between motoneurons, we accurately measured the conductance recruited by an action potential. Several difficulties had hindered accurate measurements in previous studies. (1) The depolarizing pulses used in voltage-clamp experiments (Barrett et al., 1980) do not induce the same intracellular increase in calcium as an action potential. (2) Many motoneurons exhibit a hyperpolarizationactivated $\left(I_{\mathrm{h}}\right)$ inward rectifier current (Ito and Oshima, 1965; Pape, 1996), which induces a sag in the voltage response to sustained current (Kiehn et al., 2000; Kjærulff and Kiehn, 2001) and might decrease the amplitude of the AHP and accelerate its relaxation (Gustafsson and Pinter, 1985). (3) Determining the AHP conductance from the amplitude of the voltage response to short current pulses (Ito and Oshima, 1962; Schwindt and Calvin, 1973; Baldissera and Gustafsson, 1974) was precluded by the large errors inherent to this method. To circumvent these prob- 
lems, we used a novel method by which an AHP-like current was injected in the soma via dynamic clamp. This increased the AHP amplitude. We analyzed how the intensity of the natural AHP current then was modified, allowing us to determine accurately the AHP conductance recruited by a single spike and the reversal potential of the AHP current. We showed that the conductance was not correlated with the AHP decay time and that its distribution was similar in slow- and fasttype motoneurons. Finally, we considered the functional consequences of this unexpected result.

\section{Materials and Methods}

Animal preparation

Experiments were performed on 13 adult cats (3.25-4.25 kg) deeply anesthetized with sodium pentobarbitone (pentobarbital; Sanofi, Paris, France), in accordance with French legislation. Anesthesia was induced with an intraperitoneal injection $(45 \mathrm{mg} / \mathrm{kg})$, supplemented whenever necessary by intravenous injections $(3.6 \mathrm{mg} / \mathrm{kg})$. Animals were paralyzed with pancuronium bromide (Pavulon; Organon SA, Puteaux, France) at a rate of $0.4 \mathrm{mg} / \mathrm{h}$ and artificially ventilated (end tidal $\mathrm{pCO}_{2}$ maintained at $\sim 4 \%$ ). A bilateral pneumothorax prevented movements of the rib cage. The adequacy of anesthesia was assessed on myotic pupils and on the stability of blood pressure (measured in the carotid) and of heart rate. At the onset of the experiments, amoxicillin $(500 \mathrm{mg}$; Clamoxyl; Merieux, Marcy l-Etoile, France) and methylprednisolone (5 mg; Solu-Medrol; Pharmacia, Uppsala, Sweden) were given subcutaneously to prevent the risk of infection and edema, respectively. The central temperature was kept at $38^{\circ} \mathrm{C}$. Blood pressure was maintained above 90 $\mathrm{mmHg}$ by perfusion of a $4 \%$ glucose solution containing $\mathrm{NaHCO}_{3}(1 \%)$ and gelatin (14\% Plasmagel; Roger Bellon, Neuilly-sur-Seine, France) at a rate of 3-12 ml/h. A catheter allowed for evacuation of urine from the bladder. At the end of the experiments, the animals were killed with a lethal intravenous injection of pentobarbitone $(250 \mathrm{mg})$.

The following nerves were cut, dissected, and mounted on a pair of stimulating electrodes to identify the recorded motoneurons: anterior biceps and semi-membranosus together (ABSm), posterior biceps and semi-tendinosus together (PBSt), gastrocnemius medialis together with gastrocnemius lateralis and soleus [triceps surae (TS)], the remaining part of the tibialis nerve (Tib), and the common peroneal nerve (CP). The lumbosacral spinal segments were exposed by laminectomy, and the tissues in hindlimb and spinal cord were covered with pools of mineral oil kept at $38^{\circ} \mathrm{C}$.

\section{Recordings}

We made intracellular recordings of motoneurons, using micropipettes filled with $\mathrm{KCl}$ ( $3 \mathrm{M}$; tip diameter, 2-2.5 $\mu \mathrm{m}$; resistance, 2-4 M $\Omega$ ), in discontinuous current-clamp (DCC) mode $(9 \mathrm{kHz})$ of the Axoclamp 2B (Molecular Devices, Chatillon, France). This is the recommended mode for dynamic-clamp experiments (Brizzi et al., 2004; Prinz et al., 2004) because it allows for reliable measurements of the membrane potential, even when large currents are injected. Identification of motoneurons rested on the observation of an antidromic action potential after the electrical stimulation of their axon in a peripheral nerve. We recorded the voltage response of motoneurons to a series of current pulses (intensity, -5 to $+5 \mathrm{nA}$, increasing by steps of $1 \mathrm{nA} ; 1 \mathrm{~s}$ duration, repeated at the rate of $0.5 \mathrm{~Hz}$ ). For each intensity, at least five successive traces were recorded and averaged off-line. Single action potentials were elicited by brief depolarizing pulses (duration, $1 \mathrm{~ms}$; 5-25 $\mathrm{nA}$ ) rather than by nerve stimulation, because this avoided contamination of the AHP by recurrent inhibition. A series of at least 15 control action potentials (elicited at a rate of 1 or $2 \mathrm{~Hz}$ ) was recorded and averaged.

Using a dynamic-clamp protocol, we injected an AHP-like current in the soma that added to the natural AHP current. Our dynamic-clamp setup is shown schematically in Figure $1 A$. The dynamic-clamp current (Fig. $\left.1 B_{2}\right), I_{\mathrm{dc}}=G_{\mathrm{dc}}^{\max } z(t)\left(V_{\mathrm{dc}}-V\right)$, with maximal conductance $G_{\mathrm{dc}}^{\max }$ and reversal potential $V_{\mathrm{dc}}$, was applied through the same micropipette that was used for intracellular recordings. The activation variable $z(t)$ instantaneously increased from 0 to 1 when the membrane voltage crossed the value $0 \mathrm{mV}$ and then relaxed exponentially to 0 with the time constant $\tau_{\mathrm{dc}}$ (Fig. $1 B_{1}$ ). The dynamic-clamp current did not modify the amplitude and the shape of the action potential (Fig. 1C), but it increased the amplitude of the AHP and advanced the time at which the trough of the AHP occurred (Fig. $1 B_{3}$ ).

The dynamic-clamp current was calculated by using the sequencer of the Power1401 analog-to-digital/digital-to-analog unit (Cambridge Electronic Design, Cambridge, UK). The central processing unit embedded in this unit executes one instruction every $10 \mu \mathrm{s}$. Our implementation took 11 steps (i.e., $110 \mu$ s to complete the entire loop from membrane potential sampling to current injection). This time was the same as the sampling period, which ensured a real-time feedback loop. The Power1401 unit was under the control of a personal computer (PC) running the Spike2 software (Cambridge Electronic Design). In our experiments, the reversal potential and the decay time constant of the dynamic-clamp current were always set to $-100 \mathrm{mV}$ and $23 \mathrm{~ms}$, respectively. Maximal conductance was increased up to $0.5 \mu \mathrm{S}$ by steps of $0.05 \mu \mathrm{S}$. For each conductance, at least 15 spikes were recorded. All data were stored on the PC hard drive for off-line analysis.

\section{Data analysis}

Physiological type. The averaged voltage trace after the emission of an action potential was used to measure the amplitude, duration, and halfdecay time of the natural AHP. We classified motoneurons into physiological types such as those used by Zengel et al. (1985). We used the ratio of the AHP half-decay time to the motoneuron input conductance because it was shown to predict the type correctly for $90 \%$ of the motor units. Motoneurons with a ratio of $<6$ were classified as FF type (i.e., innervating fast twitch, fatigable motor units). Those with a ratio of $>33.3$ were classified as $\mathrm{S}$ type (i.e., innervating slow twitch, fatigueresistant motor units). Those in between were FR type (i.e., innervating fast twitch, fatigue-resistant motor units). 
Subthreshold responses and estimation of the activation time constant of the $\mathrm{I}_{h}$ current. The voltage response of many motoneurons to hyperpolarizing or depolarizing current pulses exhibited a sag (Ito and Oshima, 1965; Nelson and Frank, 1967; Gustafsson and Pinter, 1984). This was likely attributable to the activation (during a hyperpolarizing pulse) or the deactivation (during a depolarizing pulse) of an $I_{\mathrm{h}}$ current (Kiehn et al., 2000). We plotted the linear relationship between the current intensity and the peak voltage response (indicated by the triangle in Fig. $2 A_{1}$ ). The reciprocal of the slope gave us the input conductance of the neuron. The $I-V$ relationship for the plateau response (indicated by the gray dot in Fig. $2 A_{1}$ ) was plotted, which allowed us to determine the plateau conductance. The "sag ratio," defined as the plateau conductance over the input conductance, then was computed. We fitted the voltage relaxation to the plateau in the response to a 3 or $4 \mathrm{nA}$ hyperpolarizing pulse with an exponential function. An estimate of the activation time constant of the $I_{\mathrm{h}}$ current was obtained by multiplying the time constant of this exponential relaxation by the sag ratio (supplemental material, available at www.jneurosci.org). This estimated time constant was systematically shorter than the true time constant at the resting potential.

Measurement of the relaxation time constant of the AHP. The relaxation time constant of the AHP conductance, $\tau_{\mathrm{AHP}}$, could be deduced readily from the voltage trace provided that $\tau_{\mathrm{AHP}}$ was at least five times smaller than the estimated activation time constant of the $I_{\mathrm{h}}$ current (supplemental material, available at www.jneurosci.org). In these conditions, a linear zone, lasting at least one-fourth of the voltage relaxation, appeared on the logarithmic plot of the AHP trajectory. An exponential was fit on the corresponding portion of the AHP, allowing us to determine $\tau_{\mathrm{AHP}}$.

Measurement of the conductance and reversal potential of the AHP current. In our method, the artificial AHP current was used to increase the AHP amplitude. This modified the intensity of the natural AHP current. We obtained a linear relationship between the AHP amplitude and the natural AHP current at the voltage trough. We derived, from the coefficients of the linear regression between these two quantities, the AHP conductance at time 0 (i.e., when the downstroke of the spike crossed the baseline level), $G_{\mathrm{AHP}}^{0}$, and the reversal potential of the AHP current relative to the resting potential, $V_{\mathrm{AHP}}$.

For each value of the artificial AHP conductance, we measured the amplitude, $\Delta V=V_{\text {rest }}-V_{\text {min }}$, of the AHP $(\Delta V>0)$ at the voltage minimum $\left(V_{\min }\right)$ and the time at which this minimum occurred $\left(t_{\min }\right)$. We then estimated the intensity of the natural AHP current at $t_{\min }$. The capacitive current vanished at the voltage trough, and the $I_{\mathrm{h}}$ current did not activate substantially compared with rest because of its slowness (supplemental material, available at www.jneurosci.org). Therefore, the total AHP current at the voltage minimum counterbalanced the leak through the somatic membrane and the dendrites. This leak was computed as the product of the input conductance and the AHP amplitude. Subtraction of the dynamic-clamp current from the leak current then gave us the intensity of the natural AHP current at the voltage trough, $I_{\mathrm{AHP}}\left[t_{\min }\right]$. This intensity was plotted against the amplitude of the AHP for each value of the dynamic-clamp-imposed conductance. The best linear fit was computed by using the least-squares method, and the slope, $\alpha$, and $y$-intercept, $\beta$, of the regression line were measured. The coefficients $\alpha$ and $\beta$ were equal, respectively, to the following: $\left\langle I_{\mathrm{AHP}}\left[t_{\min }\right] \Delta V\right\rangle-$ $\left\langle I_{\mathrm{AHP}}\left[t_{\text {min }}\right]\right\rangle\langle\Delta V\rangle\left\langle\Delta V^{2}\right\rangle-\langle\Delta V\rangle^{2}$ and $\left\langle I_{\mathrm{AHP}}\left[t_{\min }\right]\right\rangle\left\langle\Delta V^{2}\right\rangle-\left\langle I_{\mathrm{AHP}}\left[t_{\text {min }}\right] \Delta V\right\rangle$ $\langle\Delta V\rangle /\left\langle\Delta V^{2}\right\rangle-\langle\Delta V\rangle^{2}$, with the angle brackets denoting an average over the data set. We assumed that the AHP conductance decayed exponentially to 0 after a single spike (Schwindt and Calvin, 1973; Baldissera and Gustafsson, 1974; Barrett et al., 1980) (for neocortical pyramidal cells, see Abel et al., 2004) so that $I_{\mathrm{AHP}}\left[t_{\min }\right]=G_{\mathrm{AHP}}^{0} e^{-t_{\min } / \tau_{\mathrm{AHP}}}\left(V_{\mathrm{AHP}}+\Delta V\right)$. It followed that the AHP conductance recruited by a single spike, $G_{\mathrm{AHP}}^{0}$, and the reversal potential, $V_{\mathrm{AHP}}$, were given by the following equations: $c_{1} G_{\mathrm{AHP}}^{0}+c_{2} G_{\mathrm{AHP}}^{0} V_{\mathrm{AHP}}=\alpha$ and $c_{3} G_{\mathrm{AHP}}^{0}+c_{4} G_{\mathrm{AHP}}^{0} V_{\mathrm{AHP}}=\beta$, where

$$
\begin{gathered}
c_{1}=\frac{\langle\Delta V\rangle\left\langle\Delta V e^{-t_{\min } / \tau_{\mathrm{AHP}}}\right\rangle-\left\langle\Delta V^{2} e^{-t_{\min } / \tau_{\mathrm{AHP}}}\right\rangle}{\left\langle\Delta V^{2}\right\rangle-\langle\Delta V\rangle^{2}} \\
c_{2}=\frac{\left\langle\Delta V e^{-t_{\min } / \tau_{\mathrm{AHP}}}\right\rangle-\langle\Delta V\rangle\left\langle e^{-t_{\min } / \tau_{\mathrm{AHP}}}\right\rangle}{\left\langle\Delta V^{2}\right\rangle-\langle\Delta V\rangle^{2}}
\end{gathered}
$$

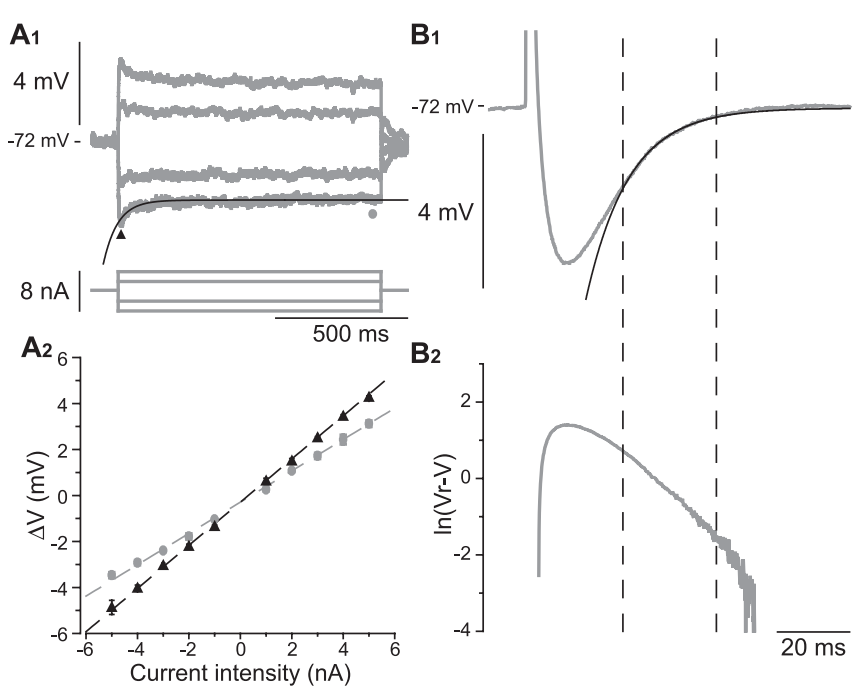

Figure 2. Differences in the passive, $A H P$, and $I_{\mathrm{h}}$ time constants. $A_{1}$, Voltage trajectories (top traces) in response to small depolarizing or hyperpolarizing steps of current (bottom traces). The time constant of the sag process was obtained by fitting an exponential function (black curves) on the averaged voltage trace (4 sweeps). It is clearly visible that the time constant depended on the amplitude of the voltage response and tended to be shorter for larger current steps. We estimated the sag time constant on the voltage trajectory (here in response to a -4 $\mathrm{nA}$ hyperpolarizing pulse) for which the peak was the closest to the AHP amplitude. $A_{2}$, The mean voltage response at the peak (filled triangles; error bars indicate SDs) was plotted against the current intensity to compute the input conductance. The plateau conductance was obtained from the plot of the mean voltage at the end of the plateau (gray dots) against the current intensity. $\boldsymbol{B}$, Measurement of the AHP time constant. $\boldsymbol{B}_{\boldsymbol{1}}$, Average trajectory (39 traces). An exponential fit (black curve) was performed on the region (between the vertical dashed lines) in which the semi logarithmic trace $\left(\boldsymbol{B}_{2}\right)$ was linear. All recordings were from the same motoneuron as in Figure 1.

$$
\begin{gathered}
c_{3}=\frac{\langle\Delta V\rangle\left\langle\Delta V^{2} e^{-t_{\min } / \tau_{\mathrm{AHP}}}\right\rangle-\left\langle\Delta V^{2}\right\rangle\left\langle\Delta V e^{-t_{\min } / \tau_{\mathrm{AHP}}}\right\rangle}{\left\langle\Delta V^{2}\right\rangle-\langle\Delta V\rangle^{2}} \\
c_{4}=\frac{\left\langle\Delta V^{2}\right\rangle\left\langle e^{-t_{\min } / \tau_{\mathrm{AHP}}}\right\rangle-\langle\Delta V\rangle\left\langle\Delta V e^{-t_{\min } / \tau_{\mathrm{AHP}}}\right\rangle}{\left\langle\Delta V^{2}\right\rangle-\langle\Delta V\rangle^{2}} .
\end{gathered}
$$

Solving this system, we obtained $G_{\mathrm{AHP}}^{0}=\left(\alpha c_{4}-\beta c_{2}\right) /\left(c_{1} c_{4}-c_{2} c_{3}\right)$ and $V_{\mathrm{AHP}}=\left(\alpha c_{3}-\beta c_{1}\right) /\left(\beta c_{2}-\alpha c_{4}\right)$.

If $t_{\text {min }}$ were the same in all conditions, we would have $c_{4}=-c_{1}=$ $e^{-t_{\min } / \tau_{\mathrm{AHP}}}, c_{2}=c_{3}=0, G_{\mathrm{AHP}}^{0}=\alpha$, and $V_{\mathrm{AHP}}=-\beta / \alpha$. The AHP conductance would be given simply by the slope of the regression line. This simple situation never occurred for motoneurons; increasing the conductance of the dynamic-clamp current advanced the voltage trough. This effect must be taken into account to estimate the AHP conductance correctly (supplemental material, available at www.jneurosci.org).

\section{Results}

We studied 44 motoneurons (18 ABSm, 13 CP, 5 PBSt, 7 Tib, and $1 \mathrm{TS}$ ) in which the resting membrane potential was lower than $-60 \mathrm{mV}$ and that displayed action potentials of $>70 \mathrm{mV}$ in amplitude. These conditions remained stable during the entire recording session.

\section{A case study}

In 33 motoneurons ( $75 \%$ of the sample), the conditions for applying our method were satisfied, as exemplified by the motoneuron shown in Figure 2. This motoneuron, ascribed to the FR type, exhibited a marked sag in response to current pulses (Fig. $2 A_{1}$ ). The input conductance was equal to $1 \mu \mathrm{S}$, and the sag ratio was equal to 1.4 (Fig. $2 \mathrm{~A}_{2}$ ). We estimated the time constant of the $I_{\mathrm{h}}$ current responsible for the sag. In response to a $4 \mathrm{nA}$ hyperpolarizing pulse, the maximum voltage response was $3.9 \mathrm{mV}$ (close to 

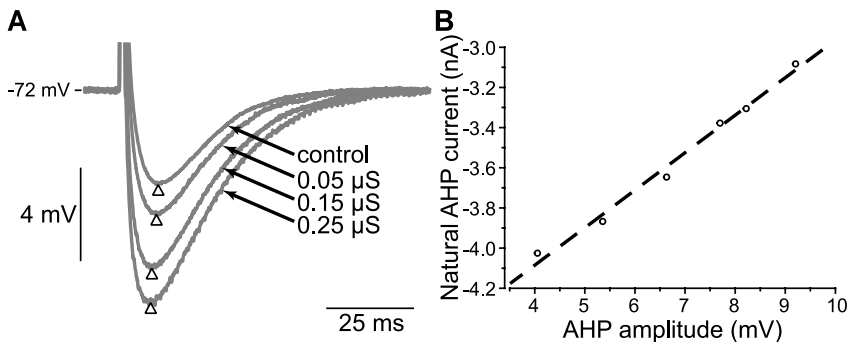

Figure 3. Measurement of the AHP conductance and reversal potential. A, Averaged AHP traces obtained without (control) and with different artificial AHP conductances. The artificial AHP current increased the AHP amplitude (open triangles) and shortened the time needed to reach the trough of the AHP. $\boldsymbol{B}$, There was a very good linear relationship $(r=0.99)$ between the natural AHP current, computed as explained in Materials and Methods, and the AHP amplitude. From the slope and the $y$-intercept of the best linear fit (dashed line) to the data points (open circles) we deduced the AHP conductance at time 0 and the AHP reversal potential (see Materials and Methods).

the AHP amplitude) (Fig. $2 B_{1}$ ). After the peak, the voltage decayed to a plateau voltage of $2.8 \mathrm{mV}$, with a time constant of $55 \mathrm{~ms}$. Multiplying this time constant by the sag ratio gave $77 \mathrm{~ms}$. This value was taken as an estimation of the $I_{\mathrm{h}}$ time constant. The semi-logarithmic plot of the voltage trajectory that followed the action potential displayed a clear linear zone lasting $22 \mathrm{~ms}$ (Fig. $2 \mathrm{~B}_{2}$, between the vertical dashed lines), amounting to $35 \%$ of the AHP relaxation, from the voltage trough to the return to the resting potential. An exponential fit of the voltage trajectory in this zone (Fig. $2 B_{1}$ ) yielded a time constant of $12 \mathrm{~ms}$ (i.e., 6.4 times less than the estimated activation time constant of the $I_{\mathrm{h}}$ current). In view of the difference between these two time constants, the voltage relaxation reflected well the exponential decay of the AHP conductance (supplemental material, available at www.jneurosci.org). Moreover, we measured the passive time constant on the off-response to brief hyperpolarizing current pulses (data not shown). It was significantly shorter $(4.5 \mathrm{~ms})$ than the AHP time constant and thereby could not affect voltage decay.

Figure 3 illustrates how we measured the AHP conductance in this motoneuron. The amplitude of the AHP that followed an action potential was $4.0 \mathrm{mV}$. Adding an AHP-like current with the dynamic clamp increased the AHP amplitude, up to $9.3 \mathrm{mV}$ when the conductance of this extra current was $0.25 \mu \mathrm{S}$ (Fig. $3 A$ ). In addition, the trough of the AHP (open triangles) occurred earlier (5.5 ms for a conductance of $0.25 \mu \mathrm{S}$ compared with 7.7 $\mathrm{ms}$ in control condition). The intensity of the natural AHP current at the minimal potential was estimated for each value of the dynamic-clamp current conductance (see Materials and Methods) and was plotted versus the AHP amplitude (Fig. 3B). A linear relationship was found $(r=0.99)$ with a slope of $0.19 \mu \mathrm{S}$ and a $y$-intercept of $-4.8 \mathrm{nA}$. From these figures and the relaxation time constant of the natural AHP (12 ms; see above), we deduced, as explained in Materials and Methods, that the natural AHP conductance was equal to $0.56 \mu \mathrm{S}$ just after the action potential. We obtained a reversal potential of $-90 \mathrm{mV}$ (i.e., $18 \mathrm{mV}$ below the resting membrane potential).

\section{AHP conductance is distributed similarly in slow and fast motoneurons}

Figure $4 A$ shows how the AHP conductance recruited by a single action potential was distributed in our sample. It ranged from 0.3 to $1.4 \mu \mathrm{S}[0.7 \pm 0.3 \mu \mathrm{S}$ (mean $\pm \mathrm{SD}) ; n=33]$. Twelve motoneurons were classified as S, 18 as FR, and three as FF (see Materials
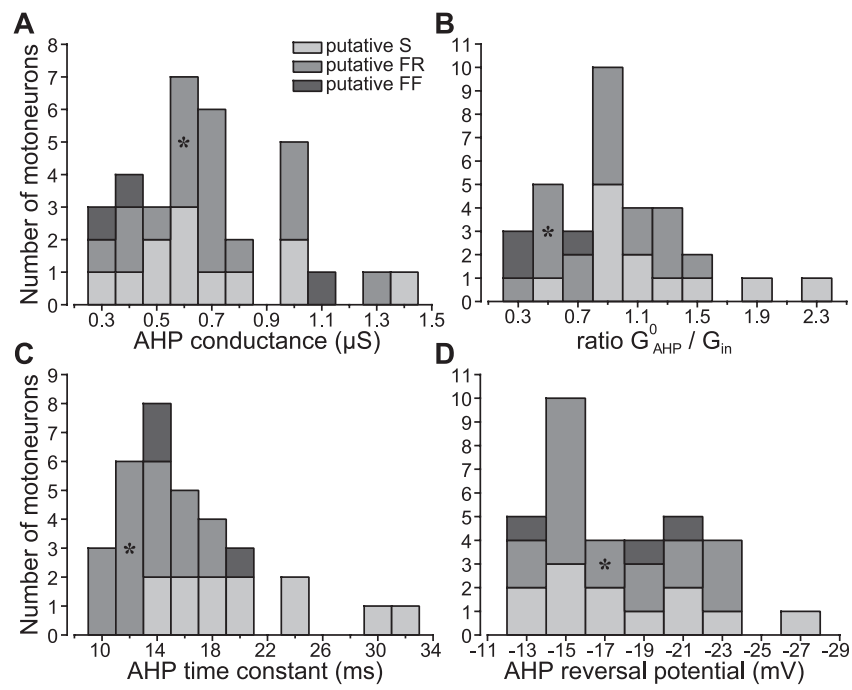

Figure 4. AHP characteristics over the entire motoneuron sample. $A$, Distribution of the AHP conductance at time 0 in the three putative physiological types ( $S$, light gray; FR, gray; FF, dark gray). $\boldsymbol{B}$, Distribution of the ratio of the AHP conductance to the input conductance. $\boldsymbol{C}$, Distribution of the AHP time constant. $D$, Distribution of the AHP reversal potential with respect to the resting potential (relative reversal potential). The asterisks indicate the motoneuron shown in previous figures.
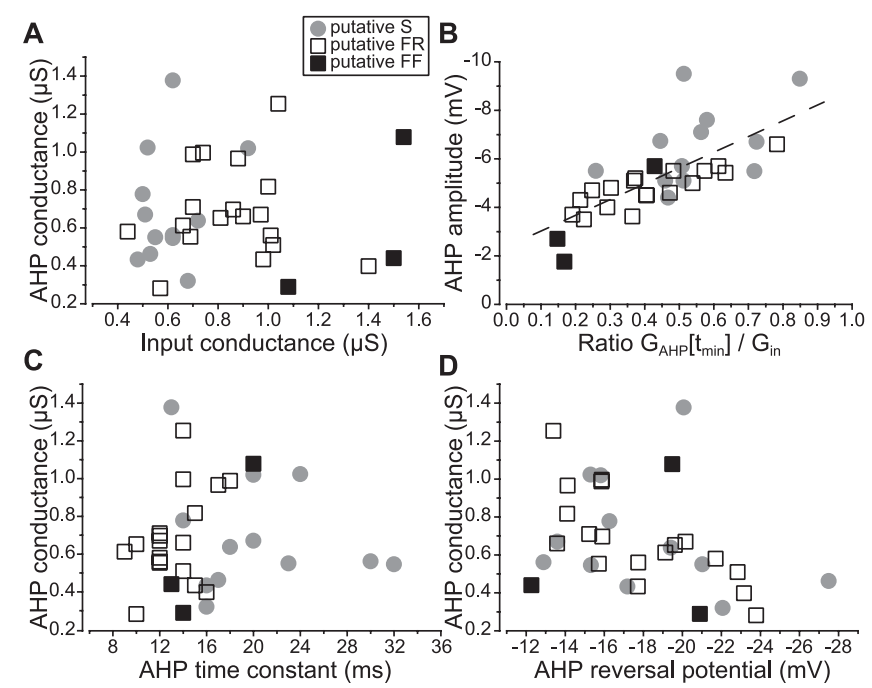

Figure 5. Lack of correlation among the different parameters. The AHP conductance was not correlated with the input conductance $(\boldsymbol{A})$, with the AHP time constant $(\boldsymbol{C})$, or with the AHP reversal potential $(\boldsymbol{D})$ regardless of the physiological type $(S$, gray dots; $F R$, open squares; $F F$, filled squares). $\boldsymbol{B}$, However, there was a clear linear correlation (dashed line; $r=0.72$ ) between the AHP amplitude and the relative AHP conductance (ratio of the AHP conductance at voltage trough to the input conductance, i.e., $\left.G_{\mathrm{AHP}}\left[t_{\min }\right]=G_{\mathrm{AHP}}^{0} e^{-t_{\min } / \tau_{\mathrm{AHP}}}\right)$.

and Methods). The AHP conductance was in the same range for all three physiological types. The average conductance was the same $(0.7 \pm 0.3 \mu \mathrm{S})$ for the slow- and fast-type (FR and FF together) motoneurons. Interestingly, the AHP conductance was distributed in the same range as the input conductance $(0.4-1.5$ $\mu S ; 0.8 \pm 0.3 \mu S ; n=33)$. However, no correlation was found between these two quantities (Fig. 5A).

AHP amplitude is controlled by the relative AHP conductance In contrast, the relative AHP conductance (i.e., the ratio of the AHP conductance to the input conductance) showed a dependence on the physiological type (Fig. 4B). The AHP conductance 
ranged from one-third to twice the input conductance (relative conductance, $0.3-2.2 ; 0.9 \pm 0.4 ; n=33)$. The mean value of the S-type motoneurons $(1.2 \pm 0.5 ; n=12)$ was significantly greater than in FF and FR motoneurons together $(0.8 \pm 0.4 ; n=21 ; p<$ 0.05 ; Student's $t$ test). This difference was attributable to the fact that the average input conductance was smaller for the slow motoneurons $(0.6 \pm 0.1 \mu \mathrm{S})$ than for the fast motoneurons (FF and FR; $0.9 \pm 0.3 \mu \mathrm{S} ; p<0.001)$. This explains why the average AHP amplitude was larger $(p<0.001)$ in slow motoneurons $(6.5 \pm$ $1.6 \mathrm{mV}$ ) than in fast ones $(4.6 \pm 1.1 \mathrm{mV}$ ) (Bakels and Kernell, 1993). Moreover, the AHP amplitude was correlated linearly with the relative AHP conductance computed at the trough of the AHP $(r=0.72)$ (Fig. $5 B)$. Altogether, our results indicate that the difference in the AHP amplitude between slow and fast motoneurons mainly results from the difference in their input conductance.

\section{AHP conductance is not correlated with the relaxation time constant}

The distribution of the relaxation time constant of the AHP conductance is shown in Figure 4C. It ranged from 9 to $32 \mathrm{~ms} \mathrm{(16 \pm}$ $5 \mathrm{~ms}$ ). This time constant was, of course, correlated with the physiological type, because the half-relaxation time was one of the criteria used to classify motoneurons (Zengel et al., 1985). Among the 17 motoneurons with an AHP time constant below 15 ms, only two were of the slow type. In contrast, 10 motoneurons among the 16 with a time constant longer than $15 \mathrm{~ms}$ were of the slow type. The mean time constant was larger in slow-type motoneurons ( $20 \pm 6 \mathrm{~ms}$ ) than in FR and FF types together (14 $\pm 3 \mathrm{~ms} ; p<$ $0.05)$. No correlation was observed between the AHP conductance at time 0 and the AHP relaxation time constant (Fig. $5 C$ ).

\section{Distribution of the AHP reversal potential}

The AHP reversal potential ranged from -27 to $-12 \mathrm{mV}$ below the resting membrane potential $(-18 \pm 4 \mathrm{mV} ; n=33)$. Figure $4 D$ shows that this relative reversal potential was distributed similarly in slow and fast motoneurons. The absolute reversal potential ranged from -68 to $-95 \mathrm{mV}(-83 \pm 7 \mathrm{mV} ; n=33)$ (histogram not shown) and also was uncorrelated with the physiological type. Just after the action potential, when the potential crossed the resting membrane potential level, the driving force of the AHP current was equal to the relative reversal potential. We did not observe any correlation between this driving force and either the AHP conductance (Fig. 5D) or the AHP relaxation time constant (data not shown). The AHP current at time 0 was obtained by multiplying the driving force by the AHP conductance. It ranged from -5 to $-27 \mathrm{nA}(-12 \pm 4 \mathrm{nA} ; n=33)$, with no statistical difference being found between slow $(-12 \pm 6$ $\mathrm{nA} ; n=12)$ and fast $(-11 \pm 4 \mathrm{nA} ; n=21)$ motoneurons.

\section{Discussion}

We measured the AHP conductance recruited by a single spike in 33 of 44 spinal motoneurons of anesthetized cats. In these motoneurons, the $I_{\mathrm{h}}$ current had a negligible impact on the amplitude and the trajectory of the AHP. Eleven motoneurons (3 S, 5 $\mathrm{FR}$, and $3 \mathrm{FF}$ ) were excluded from analysis either because the relationship between the AHP amplitude and the AHP current intensity was not linear or because the semi-logarithmic plot of the voltage trajectory did not display a clear linear zone, so determining the AHP time constant was problematic. This latter case arose when the estimated time constant of the $I_{\mathrm{h}}$ current was only three to four times the AHP decay time.

In the selected sample of motoneurons, the AHP conductance was spread widely $(0.3-1.4 \mu \mathrm{S} ; 0.7 \pm 0.3 \mu \mathrm{S})$, distributed in the same way in slow- and fast-type motoneurons, and correlated neither with the input conductance nor with the AHP relaxation time constant. This raised the issue of the impact of the AHP conductance per se on the functional properties of motoneurons.

\section{Accurate measurement of the AHP conductance}

At rest, the natural AHP conductance was activated negligibly. Indeed, blockade of the medium AHP current by apamin does not modify the input resistance significantly (Zhang and Krnjevic, 1987; Chandler et al., 1994). Krnjevic et al. (1978) showed that calcium chelation by EGTA had a lesser impact on the membrane conductance at rest than during the AHP; they interpreted the reduction of the input conductance as attributable to the action of EGTA on potassium channels other than SKs. Finally, Barrett et al. (1980) demonstrated in voltage-clamp experiments in spinal motoneurons that depolarizing pulses $>20 \mathrm{mV}$ above rest were required to induce a substantial AHP current. The intensity of this current increased with the amplitude and the duration of the pulse, suggesting that the AHP conductance depended on the exact shape of the action potential. Therefore, it is important to elicit the AHP by a genuine spike. This might be achieved by hybrid clamp (i.e., by clamping the voltage after the spike) (Lancaster and Adams, 1986). However, holding the potential at a hyperpolarized level would activate the $I_{\mathrm{h}}$ current, interfering with the AHP. Attempts were made to estimate the AHP conductance from the response to short current pulses (2-5 ms) applied during the AHP (Ito and Oshima, 1962; Schwindt and Calvin, 1973; Baldissera and Gustafsson, 1974). The voltage deflection at the end of the test pulse was compared with the deflection elicited by a control pulse applied at rest. This method suffered from systematic errors of $\sim 40 \%$. Hochman and McCrea (1994) showed that the input conductance could be deduced from the area of the voltage response to very brief pulses $(0.5 \mathrm{~ms})$. This method could not be applied to measure the AHP conductance, because this conductance varied during the pulse.

In our method, we determine the AHP conductance by analyzing how adding a known current to the AHP current alters the voltage trajectory. If the $I_{\mathrm{h}}$ current has a negligible impact on the voltage and the AHP conductance is the only active conductance at work, the AHP current counterbalances the leak current at the voltage trough and thus is proportional to the AHP amplitude. When we add an artificial AHP current, the relationship between the AHP amplitude and the intensity of the natural AHP current remains linear (supplemental material, available at www. jneurosci.org). The kinetics of the AHP conductance is nearly exponential and depends very little on voltage in the range traversed during the AHP, as demonstrated by voltage-clamp experiments (Barrett et al., 1980). One then may derive the AHP conductance at time 0 and the reversal potential from the coefficients of the linear regression.

Most recorded motoneurons exhibited an $I_{\mathrm{h}}$ current. We estimated the kinetics of this current and selected a sample (75\% of our population) in which the $I_{\mathrm{h}}$ current was slow enough not to contaminate the AHP. This showed that the short AHP of fasttype motoneurons (Burke, 1981) could not be attributed to the $I_{\mathrm{h}}$ current, contrary to the suggestion of Gustafsson and Pinter (1985). When the artificial AHP current was injected in these motoneurons, the activation of the $I_{\mathrm{h}}$ current at the voltage trough remained negligible despite the increase in AHP amplitude, because adding an artificial conductance decreased the membrane time constant and advanced the voltage trough. In contrast, when a hyperpolarizing current pulse was used to in- 
crease the AHP (seven motoneurons) (data not shown), the voltage trough was delayed, and the $I_{\mathrm{h}}$ current steadily increased with the pulse intensity. Consequently, the relationship between AHP amplitude and the estimated AHP current deviated from linearity.

In the selected motoneurons, the systematic errors in the AHP conductance were $\sim 5 \%$ and were related mainly to the estimate of the AHP decay time constant (supplemental material, available at www.jneurosci.org). Measurement errors on the membrane potential were small $( \pm 0.1 \mathrm{mV})$ because we averaged at least 15 traces. Together, the conductance was determined with an error of $\mathrm{L}<10 \%$. Errors in the relative reversal potential were attributable only to measurement errors and amounted to $<5 \%$.

The absolute reversal potential of the AHP current ( -68 to $-95 \mathrm{mV} ;-83 \pm 7 \mathrm{mV}$ ) was consistent with the Nernst potential of potassium ions. Barrett et al. (1980) reported reversal potentials less hyperpolarized ( -6 to $-20 \mathrm{mV}$ below rest) than ours $(-12$ to $-27 \mathrm{mV}$ ), possibly because of the activation of the $I_{\mathrm{h}}$ current at the most hyperpolarized holding potentials. A similar problem is encountered in current clamp when a hyperpolarizing current is injected to reverse the AHP current (Lape and Nistri, 2000). Our method does not require a strong hyperpolarization and is not plagued by this contamination issue.

\section{Functional implications}

The medium AHP current shapes the discharge of motoneurons (Kernell, 1999). During rhythmic discharge, the minimum frequency is equal to the reciprocal of the AHP duration. This duration depends strongly on the decay time constant of the AHP conductance but little on its magnitude; this explains why firing frequencies are lower in slow motor units. Several modeling studies (Kernell, 1968; Ermentrout, 1998; Wilson et al., 2004; Meunier and Borejsza, 2005) have suggested that the gain of a neuron with AHP is inversely proportional to the "slope factor" (i.e., the AHP relaxation time constant times the maximal AHP conductance achieved during repetitive discharge). Because of AHP summation, the maximal conductance is, in motoneurons, 1.8-2.7 times larger than the conductance recruited by a single spike (Baldissera et al., 1978). The present work shows that the conductance recruited by a spike is not correlated with the decay time constant and that the product of these two quantities is not statistically different for slow and fast motoneurons (data not shown). This explains why no convincing correlation between the gain and the physiological type of motoneurons has ever been reported.

What is the impact of the AHP conductance on the integration of synaptic inputs? A brief depolarizing current pulse will bring the membrane to the spiking voltage threshold if the charge transferred to the motoneuron is larger than the distance to the threshold times the capacitance. The product of these last two quantities is comparable in slow and fast motoneurons because the larger AHP amplitude of slow motoneurons is compensated by their smaller capacitance ( 4.8 vs $6.1 \mathrm{nF}$ ) (Gustafsson and Pinter, 1984). In contrast, the voltage decay after the peak of the response depends on the membrane conductance. By adding to the input conductance, the AHP conductance reduces the instantaneous membrane time constant and accelerates voltage relaxation. This effect is quite substantial during the voltage scoop that follows the emission of a spike, when the AHP conductance is large. It becomes negligible during the voltage ramp, when the AHP conductance has declined. In slow-type motoneurons, the total membrane conductance (input conductance plus AHP conductance at time 0 ) is on average $20 \%$ smaller than in fast mo- toneurons (data not shown). One may speculate that both types of motoneurons respond in the same way to a single synaptic input but that slow motoneurons are more responsive to composite inputs because of their longer integration time constant.

All things being equal, a smaller AHP conductance implies a smaller AHP amplitude and more temporal summation of synaptic inputs. In cats anesthetized with barbiturates, monoaminergic descending pathways are not activated (Crone et al., 1988; Lee and Heckman, 2000). In this preparation, only spontaneous release of neuromodulators may occur, and it should not induce a significant decrease in the AHP conductance. In contrast, activation of neuromodulatory brainstem nuclei in physiological conditions may lower the AHP conductance substantially and facilitate the expression of persistent inward currents, thus increasing membrane responsiveness in motoneurons (Hultborn et al., 2004). Finally, the broad distribution of the AHP conductance suggests that motoneurons constitute a heterogeneous population in which the responsiveness to synaptic inputs varies from one cell to the other. A similar heterogeneity was found in a previous study (Brizzi et al., 2004) in which we showed that motoneurons exhibited large differences in their sensitivity to shunting inhibition.

\section{References}

Abel HJ, Lee JCF, Callaway JC, Fohring RC (2004) Relationships between intracellular calcium and afterhyperpolarizations in neocortical pyramidal neurons. J Neurophysiol 91:324-335.

Bakels R, Kernell D (1993) Matching between motoneurone and muscle unit properties in rat medial gastrocnemius. J Physiol (Lond) 463:307-324.

Baldissera F, Gustafsson B (1974) Afterhyperpolarization conductance time course in lumbar motoneurones of the cat. Acta Physiol Scand 91:512-527.

Baldissera F, Gustafsson B, Parmiggiani F (1978) Saturating summation of the afterhyperpolarization conductance in spinal motoneurones: a mechanism for "secondary range" repetitive firing. Brain Res 146:69-82.

Barrett EF, Barrett JN (1976) Separation of two voltage-sensitive potassium currents, and demonstration of a tetrodotoxin-resistant calcium current in frog motoneurones. J Physiol (Lond) 255:737-774.

Barrett EF, Barrett JN, Crill WE (1980) Voltage-sensitive outward currents in cat motoneurones. J Physiol (Lond) 304:251-276.

Brizzi L, Meunier C, Zytnicki D, Donnet M, Hansel D, Lamotte d'Incamps B, van Vreeswijk C (2004) How shunting inhibition affects the discharge of lumbar motoneurones: a dynamic clamp study in anaesthetized cats. J Physiol (Lond) 558:671-683.

Burke RE (1981) Motor units: anatomy, physiology, and functional organization. In: Handbook of physiology, Vol II, Motor control (Brooks VB, ed), pp 345-422. Baltimore: Williams and Wilkins.

Chandler SH, Hsaio CF, Inoue T, Goldberg LJ (1994) Electrophysiological properties of guinea pig trigeminal motoneurons recorded in vitro. J Neurophysiol 71:129-145.

Crone C, Hultborn H, Kiehn O, Mazieres L, Wigstrom H (1988) Maintained changes in motoneuronal excitability by short-lasting synaptic inputs in the decerebrate cat. J Physiol (Lond) 405:321-343.

Ermentrout B (1998) Linearization of F-I curves by adaptation. Neural Comput 10:1721-1729.

Gustafsson B, Pinter MJ (1984) Relations among passive electrical properties of lumbar $\alpha$-motoneurones of the cat. J Physiol (Lond) 356:401-431.

Gustafsson B, Pinter MJ (1985) Factors determining the variation of the afterhyperpolarization duration in cat lumbar $\alpha$-motoneurones. Brain Res 326:392-395.

Hochman S, McCrea DA (1994) Effects of chronic spinalization on ankle extensor motoneurons. II. Motoneuron electrical properties. J Neurophysiol 71:1468-1479.

Hounsgaard J, Kiehn O (1989) Serotonin-induced bistability of turtle motoneurones caused by a nifedipine-sensitive calcium plateau potential. J Physiol (Lond) 414:265-282.

Hounsgaard J, Kiehn O, Mintz I (1988) Response properties of motoneu- 
rones in a slice preparation of the turtle spinal cord. J Physiol (Lond) 398:575-589.

Hultborn H, Brownstone RB, Toth TI, Gossard JP (2004) Key mechanisms for setting the input-output gain across the motoneuron pool. Prog Brain Res 143:77-95.

Ito M, Oshima T (1962) Temporal summation of after-hyperpolarization following a motoneurone spike. Nature 195:910-911.

Ito M, Oshima T (1965) Electrical behaviour of the motoneurone membrane during intracellularly applied current steps. J Physiol (Lond) 180:607-635.

Kernell D (1968) The repetitive impulse discharge of a simple neurone model compared to that of spinal motoneurones. Brain Res 11:685-687.

Kernell D (1999) Repetitive impulse firing in motoneurones: facts and perspectives. Prog Brain Res 123:31-37.

Kiehn O, KiærulffO, Tresch MC, Harris-Warrick RM (2000) Contributions of intrinsic motor neuron properties to the production of rhythmic motor output in the mammalian spinal cord. Brain Res Bull 53:649-659.

Kjærulff O, Kiehn O (2001) 5-HT modulation of multiple inward rectifiers in motoneurons in intact preparations of neonatal rat spinal cord. J Neurophysiol 85:580-593.

Krnjevic K, Puil E, Werman R (1978) EGTA and motoneuronal afterpotentials. J Physiol (Lond) 275:199-223.

Lancaster B, Adams PR (1986) Calcium-dependent current generating the afterhyperpolarization of hippocampal neurons. J Neurophysiol 55:1268-1282.

Lape R, Nistri A (2000) Current and voltage clamp studies of the spike medium afterhyperpolarization of hypoglossal motoneurons in a rat brain stem slice preparation. J Neurophysiol 83:2987-2995.

Lee RH, Heckman CJ (2000) Adjustable amplification of synaptic input in the dendrites of spinal motoneurons in vivo. J Neurosci 20:6734-6740.
Llinas R, Yarom Y (1981) Electrophysiology of mammalian inferior olivary neurons in vitro. Different types of voltage-dependent ionic conductances. J Physiol (Lond) 315:549-567.

Meunier C, Borejsza K (2005) How membrane properties shape the discharge of motoneurons: a detailed analytical study. Neural Comput 17:2383-2420.

Nelson PG, Frank K (1967) Anomalous rectification in cat spinal motoneurons and effect of polarizing currents on excitatory postsynaptic potential. J Neurophysiol 30:1097-1113.

Pape HC (1996) Queer current and pacemaker: the hyperpolarizationactivated cation currents in neurons. Annu Rev Physiol 58:299-327.

Prinz AA, Abbott LF, Marder E (2004) The dynamic clamp comes of age. Trends Neurosci 27:218-224.

Sah P (1996) $\mathrm{Ca}^{2+}$-activated $\mathrm{K}^{+}$currents in neurons: types, physiological roles, and modulation. Trends Neurosci 19:150-154.

Schwindt PC, Calvin WH (1973) Nature of conductances underlying rhythmic firing in cat spinal motoneurons. J Neurophysiol 36:955-973.

Schwindt PC, Crill WE (1984) Membrane properties of cat spinal motoneurons. In: Handbook of the spinal cord (Davidoff R, ed), pp 199-242. New York: Dekker.

Schwindt PC, Spain W, Fœhring RC, Stafstrom CE, Chubb MC, Crill WE (1988) Multiple potassium conductances and their functions in neurons from cat sensorimotor cortex in vitro. J Neurophysiol 59:424-449.

Wilson CJ, Weyrick A, Terman D, Hallworth N, Bevan MD (2004) A model of reverse spike frequency adaptation and repetitive firing of subthalamic nucleus neurons. J Neurophysiol 91:1963-1980.

Zengel JE, Reid SA, Sypert GW, Munson JB (1985) Membrane electrical properties and prediction of motor-unit type of medial gastrocnemius motoneurons in the cat. J Neurophysiol 53:1323-1344.

Zhang L, Krnjevic K (1987) Apamin depresses selectively the afterhyperpolarization of cat spinal motoneurons. Neurosci Lett 74:58-62. 